\title{
OPTICAL STUDIES OF ISOLATED NEUTRON STARS AND THEIR ENVIRONMENTS
}

\author{
Roberto P. Mignani \\ European Southern Observatory \\ rmignani@eso.org
}

\begin{abstract}
The results of optical studies of Isolated Neutron Stars (INSs), their Pulsar-Wind Nebulae (PWNe) and Pulsar Bow Shocks are reviewed and discussed.
\end{abstract}

Keywords: Pulsars, Isolated Neutron Stars, Optical

\section{Isolated Neutron Stars}

\section{The identification record}

The Isolated Neutron Stars (INSs) with an associated optical counterpart, including both rotation-powered pulsars and the so-called X-ray Dim INSs (XDINSs),

\begin{tabular}{l|lll|lll|l}
\hline Name & Year & Tel & Size & mag & $d(k p c)^{*}$ & $A_{V}$ & Identification \\
\hline Crab & 1969 & Steward & $0.9 \mathrm{~m}$ & 16.6 & 1.73 & 1.6 & Pulsations \\
Vela & 1976 & CTIO & $4 \mathrm{~m}$ & 23.6 & 0.23 & 0.2 & Pulsations \\
B0540-69 & 1984 & CTIO & $4 \mathrm{~m}$ & 22 & 49.4 & 0.6 & Pulsations \\
Geminga & 1987 & CFHT & $3.6 \mathrm{~m}$ & 25.5 & 0.16 & 0.07 & Proper Motion/Pulsations \\
B0656+14 & 1994 & NTT & $3.5 \mathrm{~m}$ & 25 & 0.29 & 0.09 & Pulsations/Proper Motion \\
B0950+08 & 1996 & HST & $2.4 \mathrm{~m}$ & 27.1 & 0.26 & 0.03 & Position/Photometry \\
B1929+10 & 1996 & HST & $2.4 \mathrm{~m}$ & 25.6 & 0.33 & 0.15 & Proper Motion \\
B1055-52 & 1997 & HST & $2.4 \mathrm{~m}$ & 24.9 & 0.72 & 0.22 & Position \\
RXJ1856-3754 & 1997 & HST & $2.4 \mathrm{~m}$ & 25.7 & 0.14 & 0.12 & Proper Motion \\
J0720-3125 & 1998 & Keck & $10 \mathrm{~m}$ & 26.7 & & 0.30 & Proper Motion \\
B1509-58 & 2000 & VLT & $8.2 \mathrm{~m}$ & 25.7 & 4.18 & 5.2 & Position \\
RXJ1308.6+2127 & 2002 & HST & $2.4 \mathrm{~m}$ & 28.6 & & 0.14 & Position \\
RXJ1605.3+3249 & 2003 & HST & $2.4 \mathrm{~m}$ & 26.8 & & 0.06 & Proper Motion \\
J0437-4715 & 2004 & HST & $2.4 \mathrm{~m}$ & & 0.14 & 0.11 & Spectroscopy \\
\hline
\end{tabular}

${ }^{*}$ http://rsd-www.nrl.navy.mil/7213/lazio/ne_model/

Table 1. INSs identification status. The columns give the name, the year of the proposed identification, the used telescope and its aperture, the magnitude ( $V$-band when available), the distance, the interstellar absorption $A_{V}$ and the identification evidence. 
are now 14 (Table1; see also Mignani et al. 2004a) i.e. about as many as those detected in X-rays in the pre-ROSAT era. Mostly thanks to the HST and the high UV sensitivity of the FOC and the STIS, the initial identification score of two objects per decade has increased to almost one per year. As a matter of fact, in the last 10 years HST has detected all the INSs it was targetted to. On the other hand, large ground-based telescopes like the Kecks and the VLT so far played only a marginal role. Recent HST observations might have identified also the counterpart of the young 16 ms pulsar PSR J0537-6910 (Mignani et al. 2004b). Owing to their intrinsic faintness, most INSs have been detected only because of their close distance and small interstellar absorption. This made also possible to use proper motion as an alternative, and indeed very efficient, identification technique to optical timing.

\begin{tabular}{|c|c|c|c|c|c|c|}
\hline$\overline{\text { Name }}$ & Spec.(A) & Phot. & $\alpha$ & $T$ & Comments & Pol. $^{* *}$ \\
\hline$\overline{\mathrm{Crab}}$ & $1100-9000$ & UV,UBVRI,JHK & -0.11 & - & $P L_{o}<P L_{x}$ & $\begin{array}{l}20 \% \text { (IP) } \\
40 \% \text { (OP) }\end{array}$ \\
\hline B1509-58 & & $\mathrm{R}$ & & & & $10 \%(\mathrm{TI})$ \\
\hline B0540-69 & $2500-5500$ & UBVRI & +0.2 & - & $P L_{o}<P L_{x}$ & $5 \%(\mathrm{TI})$ \\
\hline Vela & $4500-8600$ & UV,UBVRI,JH & +0.12 & - & $P L_{o} \sim P L_{x}$ & $8.5 \%(\mathrm{TI})$ \\
\hline$\overline{\mathrm{B} 0656+14}$ & & UV,UBVRI,JHK & +0.45 & 8.5 & $\begin{array}{l}P L_{o} \sim P L_{x} \\
B B_{o} \sim B B_{x}\end{array}$ & $100 \%$ (IP) \\
\hline Geminga & $3700-8000$ & UV,UBVRI,JH & +0.8 & 4.5 & $\begin{array}{l}P L_{o}<P L_{x} \\
B B_{o} \sim B B_{x}\end{array}$ & \\
\hline B1055-52 & & $\mathrm{U}$ & & & & \\
\hline$\overline{\mathrm{B} 1929+10}$ & & UV,U & +0.5 & - & $P L_{o}<P L_{x}$ & \\
\hline B0950+08 & & U,BVI & +0.65 & - & $P L_{o} \sim P L_{x}$ & \\
\hline$\overline{J 0437-4715}$ & $1150-1700$ & & - & $\overline{1.0}$ & $B B_{o}>B B_{x}$ & \\
\hline$\overline{\text { RXJ0720-3125 }}$ & & $\overline{U V, U B V R}$ & $\begin{array}{ll}-1.4 \\
\end{array}$ & 4 & $B B_{o}>B B_{x}$ & \\
\hline RXJ1856-3754 & $3600-9000$ & UV,UBV & - & 2.3 & $B B_{o}>B B_{x}$ & \\
\hline RXJ1605.3+3249 & & VR & & & $B B_{o}>B B_{x}$ & \\
\hline RXJ1308.6+2127 & & $\mathrm{V}$ & & & $B B_{o}>B B_{x}$ & \\
\hline
\end{tabular}

${ }^{* *} \mathrm{IP}=$ Inter Pulse; OP=Off Pulse; TI=Time Integrated

Table 2. Optical INS database grouped by age decades. The columns give the name, spectroscopy, photometry, the spectral index $\alpha$ and temperature (in units of $10^{5} \mathrm{~K}$ ) of the power-law and blackbody components $\left(P L_{o} ; B B_{o}\right)$ and the comparison with the optical extrapolation of the X-rays ones $\left(P L_{x} ; B B_{x}\right)$. Polarization measures are indicated in the last column.

\section{Photometry and Spectroscopy}

Table 2 summarizes the optical INSs database (see also Mignani et al. 2004a and references therein). Only for six of them optical/UV spectroscopy is available and only for four photometry spans all the way from the IR to the UV. This is crucial to identify thermal and non-thermal spectral components whose contributions are expected to be markedly different in the IR and in the UV. As 
a general trend, the spectrum grows in complexity with the age from a single power-law (PL) dominated to a composite one featuring both PL and blackbody (BB) components. While in some cases the optical PL/BB components do match the extrapolation of the X-ray ones, apparently this is not a general rule, which suggests that the optical and X-ray emission mechanisms are not always related to each other. In particular, for XDINs the optical BB spectrum appears to be systematically above the extrapolation of the X-ray one. Apart from the decrease of the temperature of the $\mathrm{BB}$ component, which follows from the cooling of the neutron star surface, there is no clear indication for an evolution of the spectral parameters with the neutron star's age.

\section{Timing}

After the "historical" optical pulsars Crab, Vela and PSR B0540-69, pulsations have been clearly detected from Geminga (Romani \& Pavlov, in preparation) and PSR B0656+14 (Gull et al., 2004) thanks to recent HST/STIS observations, confirming and improving the earlier results of Shearer et al. (1997) and Shearer et al. (1998). In all cases but PSR B0540-60 the lightcurves are double-peaked and for both the Crab and Geminga the peaks are aligned in phase with the $\gamma$-ray ones.

\section{Polarimetry}

Till recently, the only INS with measured optical polarization was the Crab (see also Kamback et al., these proceedings). The breacktrough came with the VLT which measured the time-integrated polarization for PSR B0540-69, Vela and PSR B1509-58 (Wagner \& Seifert 2000). More recently, time-resolved polarization was measured for PSR B0656+14 (Kern et al. 2003).

\section{Pulsar-Wind Nebulae}

So far, Pulsar Wind Nebulae (PWNe) have been detected in the optical only for two young pulsars: the Crab (e.g., Hester et al. 2002) and PSR B0540-69 (Caraveo et al. 2000). In both cases, HST observations have clearly resolved the counterparts of the X-ray structures detected by Chandra. The PWN around the Vela pulsar originally claimed by Ogelman et al. (1989) was not confirmed by HST observations (Mignani et al. 2003) which put $3 \sigma$ upper limits of $\approx 27.9$ and $\approx 28.3-27.8$ mag $\operatorname{arcsec}^{-2}$ on the brightness of the inner and outer X-ray PWN, respectively, i.e. close the extrapolation of the X-ray/radio data. Comparable deep upper limits were set on the optical emission of the PWN around PSR J0537-6910 (Mignani et al. in preparation). 


\section{Pulsar Bow-Shocks}

The interaction between the pulsar's relativistic wind and the ISM compressed by the pulsar supersonic motion originates a shock which ionizes the ISM and produce emission in $H_{\alpha}$. The $H_{\alpha}$ luminosity $\left(L_{H_{\alpha}}\right)$ depends on the pulsar's rotational energy loss $(\dot{E})$, on the neutron star's velocity $\left(v_{N S}\right)$ and on the fraction $X$ of neutral Hydrogen in the ISM. The bow-shock shape tends to be symmetric wrt the pulsar proper motion $(\mu)$ direction, with deviations determined by the local ISM density distribution, featuring either arc-like or bullet-like structures according to the perspective. So far, optical bow-shocks have been clearly identified around 6 INSs (see Table 3). By imposing pressure balance between the (radial) pulsar wind and the ISM and assuming a geometrical model for the bow-shock one can derive the local ISM density $\left(\rho_{I S M}\right)$ and the angle of the pulsar's velocity vector wrt the line of sight. From the 3-D velocity, the distance and the age one can then trace back the galactic orbital motion of the pulsar, given a galactic potential model, and localize its birth place, hence identify its progenitor stellar population.

\begin{tabular}{l|ll|lll|l|l}
\hline Name & $\begin{array}{l}\text { Log(Age) } \\
(\mathrm{yrs})\end{array}$ & $\log (E)$ & $\begin{array}{l}\mu \\
(\mathrm{mas} / \mathrm{yr})\end{array}$ & $\begin{array}{l}d \\
(\mathrm{kpc})\end{array}$ & $\begin{array}{l}v_{N S} \\
(\mathrm{~km} / \mathrm{s})\end{array}$ & $\log \left(L_{H a}\right)$ & Comment \\
\hline B0740-28 & 5.20 & 35.14 & 29 & 1.9 & 204 & $\sim 29$ & \\
B2224+65 & 6.05 & 33.08 & 182 & 2 & 1700 & $\sim 30$ & \\
B1957+20 & 9.18 & 35.20 & 30.4 & 1.53 & 225 & $\sim 31$ & ms, binary \\
J0437-4715 & 9.2 & 34.07 & 141 & 0.14 & 98 & $\sim 28$ & ms, binary \\
J2124-3358 & 9.8 & 33.63 & 52.6 & 0.27 & 72 & $\sim 27$ & ms, isolated \\
RXJ1856-3754 & $?$ & $?$ & 333 & 0.14 & 220 & $\sim 26$ & \\
\hline
\end{tabular}

Table 3. INSs with $H_{\alpha}$ bow-shocks. Luminosities and $\dot{E}$ are in units of $\mathrm{erg}_{\mathrm{cm}}^{-2} \mathrm{~s}^{-1}$.

\section{References}

Caraveo, P.A. et al., 2000, Proc. of A decade of HST science, Eds. M. Livio, K. Noll, and M. Stiavelli, p.9

Gull, T. et al. 2004, Proc. IAU Symposium 218 "Young Neutron Stars and Their Environments", eds F. Camilo and B. M. Gaensler

Kern, B. et al., 2003 ApJ 597, 1049

Hester, J. J. et al. 2002, ApJ, 577, L49

Mignani, R.P. et al. 2004, Proc. IAU Symp. 218 "Young Neutron Stars and Their Environments", eds F. Camilo and B. M. Gaensler (astro-ph/0311468)

Mignani, R.P., et al. 2004a, submitted to A\&A

Ogelman, H. B, Koch-Miramond, L., Aurieere, M. 1989, ApJ, 342, 83

Shearer, A. et al. 1997, A\&A, 487, L181

Shearer, A. et al. 1998, ApJ 335, L21 
Wagner, S.J. \& Seifert, W., 2000, Proc. of IAU Coll. 177 "Pulsar Astronomy: 2000 and Beyond",ASP Conference Series, Vol. 202, p. 315, Eds. M. Kramer, N. Wex, and N. Wielebinski 